\title{
Archaeonautica
}

Archaeonautica

L'archéologie maritime et navale de la préhistoire à

l'époque contemporaine

$20 \mid 2018$

De re navali : Pérégrinations nautiques entre Méditerranée et océan Indien

\section{Une nouvelle maquette de restitution hypothétique d'un navire à dolia}

A new hypothetical reconstruction model of a dolia ship

\section{Robert Roman}

\section{(2) OpenEdition}

Journals

Édition électronique

URL : http://journals.openedition.org/archaeonautica/392

DOI : 10.4000/archaeonautica.392

ISSN : 2117-6973

Éditeur

CNRS Éditions

Édition imprimée

Date de publication : 6 décembre 2018

Pagination : 233-237

ISBN : 978-2-271-12263-6

ISSN : 0154-1854

Référence électronique

Robert Roman, " Une nouvelle maquette de restitution hypothétique d'un navire à dolia »,

Archaeonautica [En ligne], 20 | 2018, mis en ligne le 30 avril 2020, consulté le 30 avril 2020. URL :

http://journals.openedition.org/archaeonautica/392 ; DOI : https://doi.org/10.4000/archaeonautica. 392 


\title{
UNE NOUVELLE MAQUETTE DE RESTITUTION HYPOTHÉTIQUE D'UN NAVIRE À DOLIA
}

\author{
Robert ROMAN
}

\begin{abstract}
Résumé
La construction pour le musée d'Hyères d'une maquette échelle $1 / 10^{e}$ d'un navire à dolia, nous a permis de proposer des nouvelles hypothèses sur l'agencement à l'intérieur de la cale de ces conteneurs, sur la position du mât, ainsi que sur les formes transversales de la coque. La démarche adoptée et les résultats principaux de ce travail sont présentés dans cette contribution.
\end{abstract}

\section{Mots-clés}

Dolium, Maquette, Navire

\section{A NEW hypothetical ReCONSTRUCtion MODEl OF A DOLIA SHIP}

\section{Abstract}

The construction of a $1 / 10^{\text {th }}$ scale model of a dolia ship for the Hyères museum has led to the proposal of new theories regarding the interior arrangement of these containers in the hold, the position of the mast, as well as the transversal shape of the hull. The approach adopted and the main results of this work are presented in this contribution.

Mots-clés

Dolium, Model, Ship
La commande d'une maquette d'un navire à dolia au $1 / 10^{\mathrm{e}}$ par le Musée gallo-romain de Lyon en $2004^{1}$ nous avait permis de tenter une étude expérimentale afin de mieux comprendre la morphologie et la conception de ce type de navires (Carre, Roman 2008).

Seule l'épave à dolia de Ladispoli (Carre 1993), qui présentait alors le fond de carène le mieux conservé de toutes les épaves à dolia connues, avait pu être utilisée pour cette reconstitution. Malgré la différence de chronologie - le naufrage date des années 100 av. J.-C. - et le fait qu'il ne s'agisse pas d'un navire à dolia, les données de l'épave de Cavalière, qui avait fait l'objet d'une restitution fiable (Roman 1997, p. 78-97), ont été intégrées avec celles de l'épave de Ladispoli pour pallier la faiblesse des vestiges de cette dernière. L'ensemble du plan original avait ensuite été augmenté de façon homothétique (soit $25 \%$ ) pour être conforme aux dimensions requises par le chargement de dolia et d'amphores, disposé selon le schéma hypothétique proposé pour l'épave Grand Ribaud D (Hesnard et al. 1988, pl. XLIX et L). Au terme de ce travail nous avons obtenu un navire hypothétique de $16 \mathrm{~m}$ de long et $6 \mathrm{~m}$ de large (rapport $\mathrm{L} / 1=2,66)$.

Malgré le peu d'éléments disponibles pour cette première étude, un certain nombre d'observations avaient pu être faites : la présence d'une seule cabine à l'avant, d'une cloison séparant les gros conteneurs des amphores et le chargement de ces dernières selon un schéma en carré plutôt qu'en quinconce. Cette étude a permis également de préciser que, compte tenu des dimensions et du poids des dolia, leur mise en place n'était possible qu'avant le début du barrotage et donc préalablement à la fermeture du navire par le pont et les passavants. Ces navires particuliers devaient donc être construits non loin des ateliers à dolia ${ }^{2}$.

1. Maquette réalisée à l'occasion de l'exposition Le vin. Nectar des dieux, Génie des hommes (Brun et al. 2004).

2. Sur la question de la localisation des ateliers, voir en dernier lieu Carre à paraître et Cibecchini à paraître.
Cependant, à l'issue de la construction de la maquette, le plan de forme de l'épave de Cavalière, avec ses murailles évasées, s'est révélé incompatible avec l'arrimage des dolia dans le plan transversal. Nous avons aussi constaté que la cale n'était pas dimensionnée pour loger quatre dolia dans le plan longitudinal, selon l'hypothèse de chargement proposé pour le navire Grand Ribaud D.

\section{LA NOUVELLE MAQUETTE}

La commande par la ville d'Hyères d'une nouvelle maquette de navire à dolia nous a offert l'opportunité de reprendre la première étude.

Deux hypothèses ont alors été émises : soit les charpentiers concevaient la carène du navire en fonction du nombre de dolia et doliola soit, à l'inverse, les dolia étaient fabriqués à partir de l'espace disponible dans la cale. Dans tous les cas, il apparaît évident que le chantier naval devait travailler en lien étroit avec le fabricant des dolia de façon à construire un bateau bien équilibré et en mesure de répondre aux contraintes d'un chargement pondéreux à l'arrimage contraignant. En effet, le calage des dolia entre les flancs du navire devait être le plus ajusté possible pour supprimer les mouvements latéraux et éviter la cassure des conteneurs.

Dans la première maquette, seuls des dolia ronds avaient été placés dans la cale. Pour cette deuxième hypothèse, nous proposons un nouveau schéma de chargement utilisant également des doliola, pratique attestée sur plusieurs épaves et en particulier sur celle de Ladispoli, qui est, comme on l'a déjà souligné, le navire à dolia offrant les vestiges de coque les mieux conservés. Un plan de forme a ainsi été tracé autour de cette nouvelle cargaison hypothétique de dolia et doliola, puis replacé graphiquement sur les vestiges de l'épave de Ladispoli. 


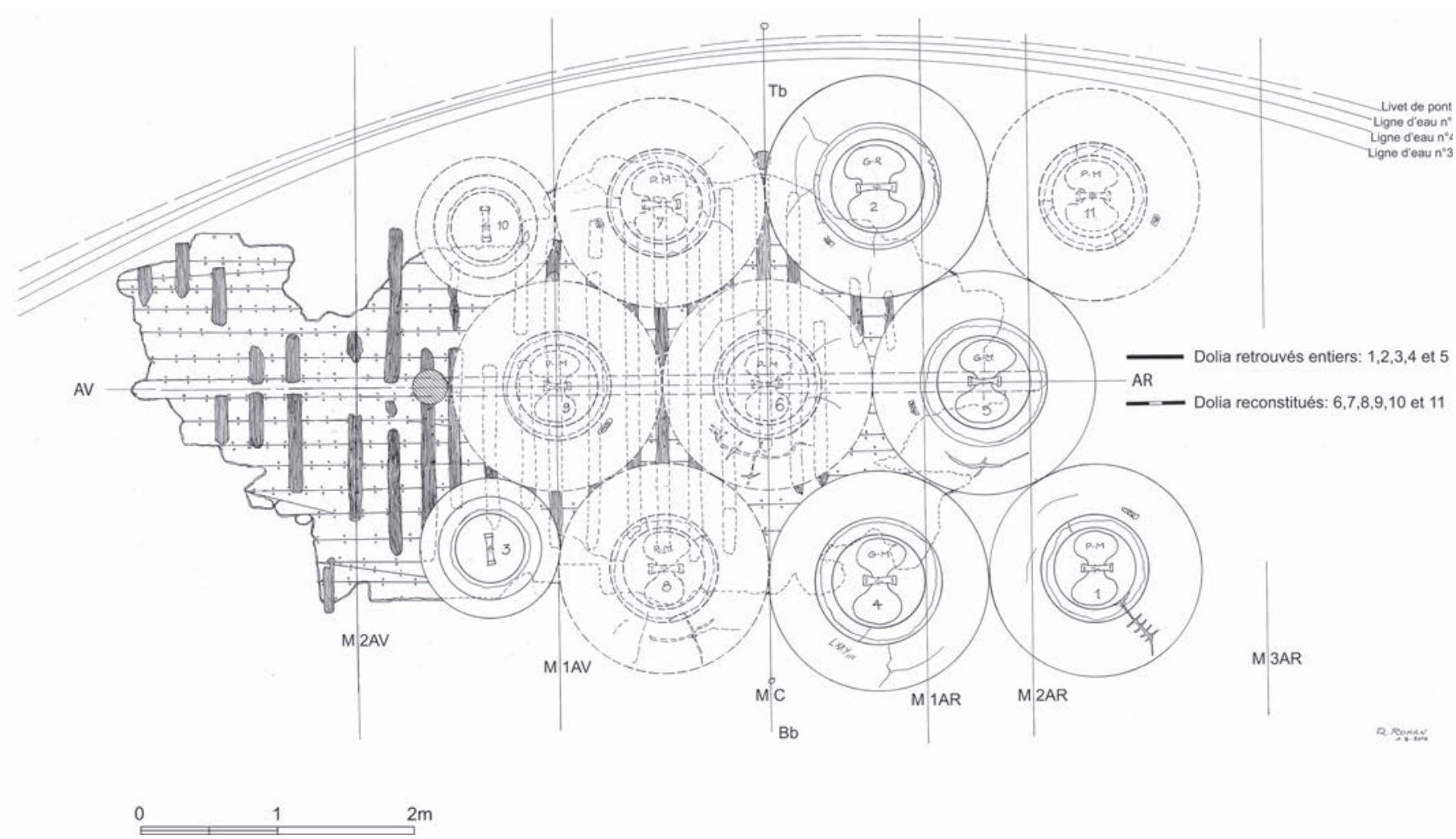

Fig. 1: Mise en place des dolia et doliola sur les vestiges archéologiques de l'épave de Ladispoli (dessin R. Roman).

Trois types de conteneurs composent ce chargement théorique : les grands modèles (diam. 1,60 à 1,70 m) et les petits modèles (diam. 1,50 m, h. 1,60 m) du Grand Ribaud D et enfin le doliolum cylindrique (diam. $1 \mathrm{~m}, \mathrm{~h} .1,70 \mathrm{~m}$ ) retrouvé à Ladispoli. Les dolia ronds sont disposés en quinconce en trois rangées, dont la rangée centrale est disposée le long de l'axe du navire, sur la carlingue/emplanture du mât. Les deux doliola sont positionnés à l'avant des deux rangées latérales (fig. 1 et 2). Ce plan de forme donne à voir, au maître-couple, des murailles droites qui calent les dolia et doliola dans le plan transversal (fig. 3). La longueur restituée reste celle de la première hypothèse $(16 \mathrm{~m})$ pour une largeur qui se réduit à $5 \mathrm{~m}$ et un rapport L/1 de 3,2.

Le plan de bordé, pour les parties non conservées a été obtenu après avoir repéré les largeurs des virures sur les vestiges du fond de carène de Ladispoli. Les parties manquantes ont été restituées en prolongeant vers l'avant et vers l'arrière ces mêmes virures. Leurs extrémités, légèrement diminuées en largeur, prennent place dans une râblure sur l'étrave pour l'avant et sur l'étambot pour l'arrière.

Pour obtenir une répartition homogène des virures et réduire la surface du bordé sur le plan transversal de manière à l'adapter aux formes du navire, six joints perdus sur chaque bord ont été insérés ${ }^{3}$. Deux préceintes, habituellement présentes dans les navires romains, ont été également rajoutées. L'aile de protection de l'appareil de gouverne prend appui sur la préceinte basse

3. À titre d'exemple, les joints perdus sont présents sur navires Napoli A (Boetto et Poveda dans ce volume) et Napoli $C$ datés de la fin du premier siècle de notre ère (Boetto 2005 ; Poveda 2012 ; Boetto, Poveda 2014). et sur la lisse de pavois (fig. 4). La phase finale de l'élaboration de la maquette tient compte des vestiges archéologiques du navire Laurons 2, bien conservée sur l'un de ses flancs jusqu'au pavois et qui avait donné lieu à une étude architecturale complète (Gassend et al. 1984).

En conservant un rapport L/l propre aux navires de commerce de l'Antiquité tout en prenant en compte l'organisation du chargement, nous obtenons un navire relativement étroit, aux murailles droites, ce qui permet de caler transversalement les lourds conteneurs et de stabiliser le chargement (fig. 3 et 5). On peut constater également l'avancée notable de la position du mât en adéquation avec la présence dans la cale d'une rangée de quatre dolia. Cette nouvelle organisation de la cargaison déplace donc la cloison de séparation entre les dolia et le chargement d'amphores vers la proue, et avec elle le pied de mât qui se retrouve ainsi au tiers avant (fig. 4). Enfin, cet arrangement détermine une nouvelle répartition des amphores, dont le nombre se trouve diminué.

Dans l'attente de nouvelles découvertes nous permettant de mieux connaître l'architecture des navires à dolia, cette étude fait ressortir des caractéristiques importantes qui, devaient avoir un certain poids lors de la conception de la structure et des formes de la carène. Il est probable que les charpentiers concevaient un navire ayant une faible largeur au maître couple avec des murailles relativement verticales, qui permettaient de se passer du calage des dolia entre le bordé et ces dernières, et avec un mât avancé. 

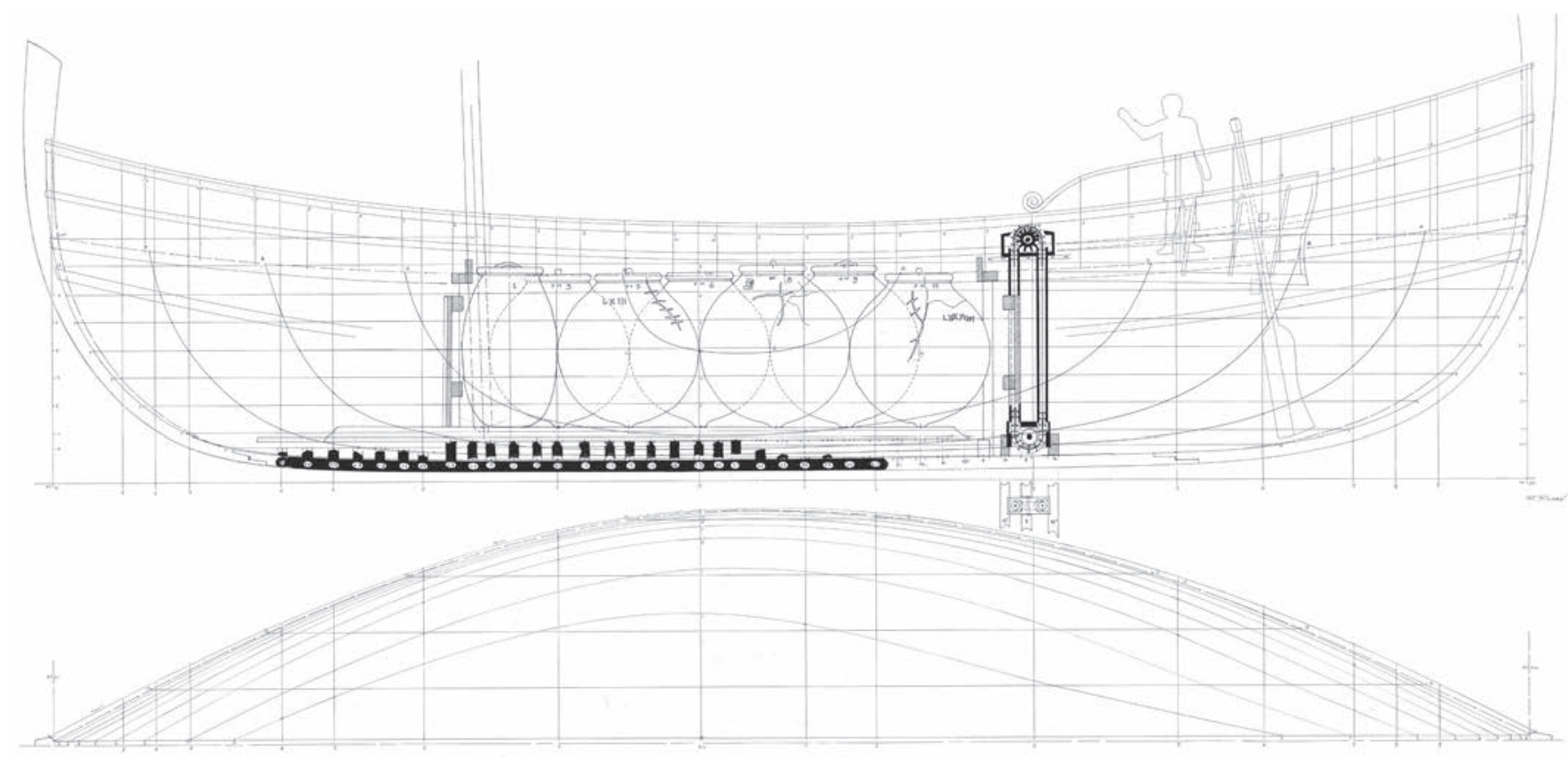

0 $5 \mathrm{~m}$

Fig. 2: Plan de forme (vues de côté et de dessus) avec le chargement, le système de gouverne et de propulsion ainsi que la pompe de cale en place (dessin R. Roman).

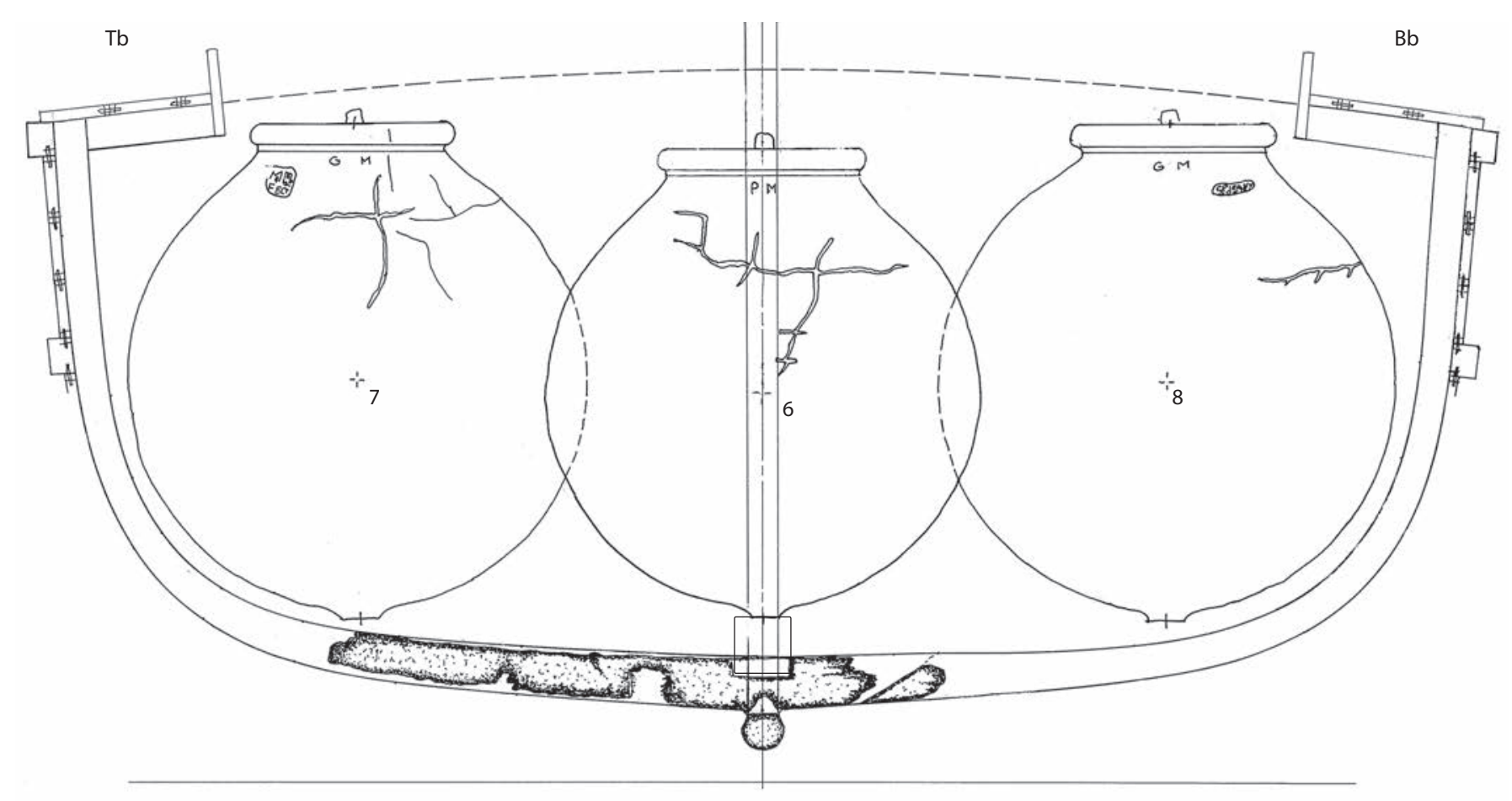

\begin{tabular}{lll}
0 & 1 \\
\hline \hline
\end{tabular}

Fig. 3: Coupe transversale au niveau du maitre-couple (varangue M19) (dessin R. Roman). 


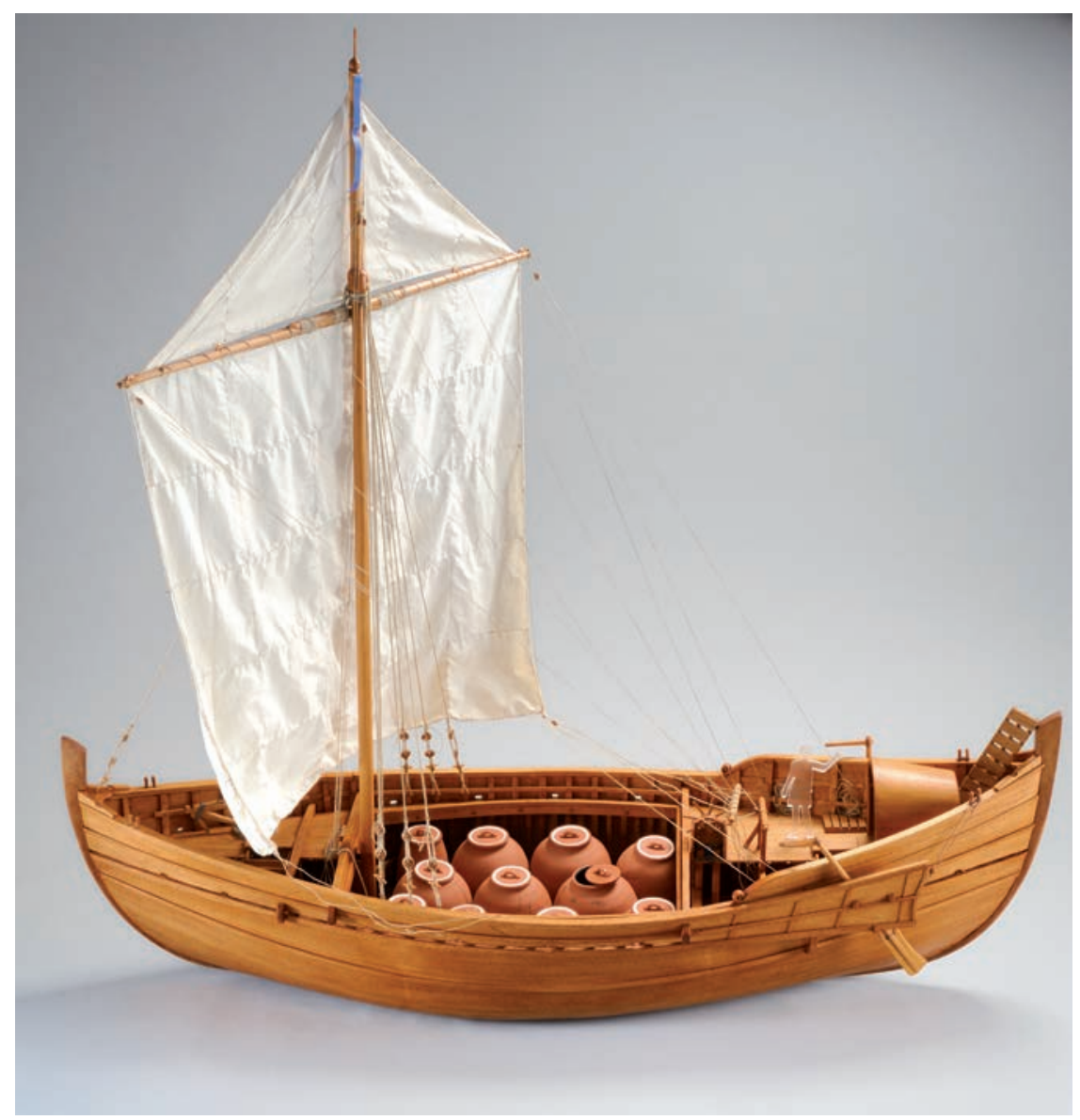

Fig. 4 : La nouvelle maquette de navire à dolia à l'échelle 1/10 (réalisation R. Roman, cliché L. Damelet, AMU/CNRS/CCJ).

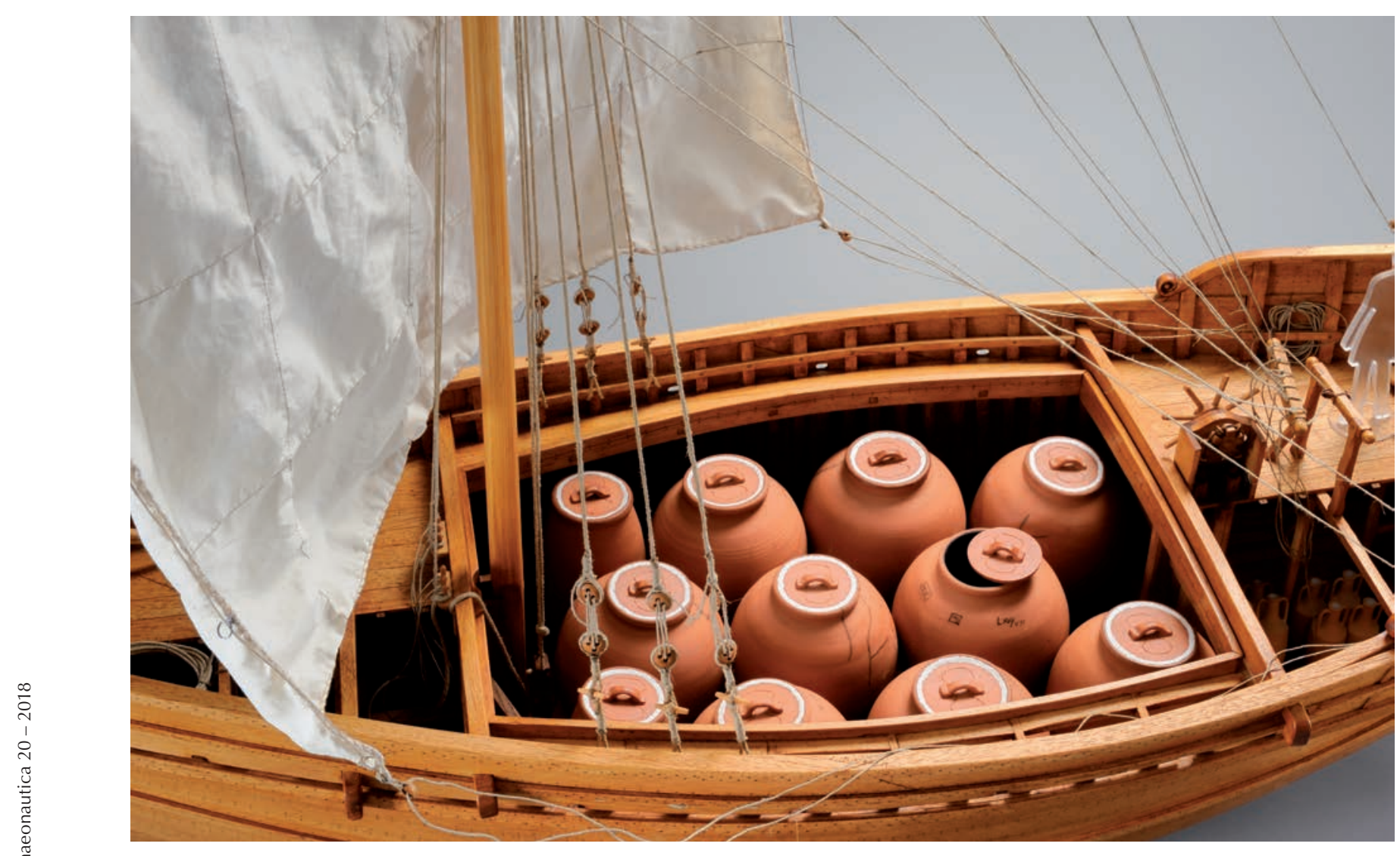

Fig. 5: Vue de la cale avec le chargement des dolia (réalisation R. Roman, cliché L. Damelet). 


\section{BIBLIOGRAPHIE}

Boetto G.

2005 Le navi romane di Napoli, dans Giampaola D. et al., La scoperta del porto di Neapolis: dalla ricostruzione topografica allo scavo e al recupero dei relitti, Archaeologia Maritima Mediterranea, 2, p. 63-76.

Boetto G., Poveda P.

2014 La restitution de Napoli $C$, un navire romain à tableau, dans P. Pomey (dir.), Ports et navires dans l'Antiquité et à l'époque byzantine, Dossiers d'Archéologie, 364, Juillet-Août, p. 64-65.

Brun J.-P., Poux M., Tchernia A. (DIr.)

2004 Le Vin. Nectar des dieux, Génie des Hommes, Gollion, Infolio.

\section{CARre M.-B}

1993 L'épave à dolia de Ladispoli (Étrurie Méridionale). Étude des vestiges de la coque, Archeonautica, 11, p. 9-29.

À paraître Les propriétaires des ateliers de dolia de Minturnes, dans C. Carrato, F. Cibecchini (dir.), Nouvelles recherches sur les dolia. L'exemple de la Méditerranée nord-occidentale à l'époque romaine ( $I^{e r}$ s. av. J.-C.- III e s. ap. J.-C.), Actes de la Table ronde, Aspiran, 2013.

\section{Carre M.-B., Roman R.}

2008 Hypothèse de restitution d'un navire à dolia : la construction de la maquette, Archaeonautica 15, p. 175-192.

\section{Cibecchini F.}

À paraître L'épave Ouest-Giraglia 2 : nouvelles épaves et nouvelles données sur le commerce du vin en vrac en Méditerranée occidentale, dans C. Carrato, F. Cibecchini (dir.), Nouvelles recherches sur les dolia. L'exemple de la Méditerranée nord-occidentale à l'époque romaine ( $I^{e r}$ s. av. J.-C.- III ${ }^{e}$ s. ap. J.-C.), Actes de la Table ronde, Aspiran, 2013.

Gassend J.-M., Liou B., XiménÈs S.

1984 L'épave 2 de l'anse des Laurons (Martigues, Bouches-du-Rhône), Archaeonautica, 4, p. 76-105.

Hesnard A., Carre M.-B., Rival M., Dangréaux B.

1988 L'épave romaine Grand Ribaud D, Paris, éditions du CNRS (Archaeonautica, 8).

Poveda P.

2012 Le navire antique comme instrument du commerce maritime : restitutions $3 D$, tonnage, qualités nautiques et calculs hydrostatiques des épaves : Napoli A, Napoli C, Dramont E et JulesVerne 7, thèse de doctorat, sous la dir. de P. Pomey, Aix-Marseille Université, Aix-en-Provence [non publiée].

ROMAN R.

1997 Étude architecturale comparative de sept navires de commerce gréco-romains et byzantins, thèse de doctorat, sous la dir. de A. Tchernia, Université de Provence, Aix-en-Provence [thèse non publiée]. 